\title{
Effects of Interior Bezel Size and Configuration on Gaming Performance with Large Tiled Displays
}

\author{
Geneva Smith*, Robert J. Teather*, Jordan Lass ${ }^{\dagger}$, Jacques Carette* \\ *Computing \& Software, ${ }^{\dagger}$ Psychology, Neuroscience \& Behaviour \\ McMaster University \\ Hamilton, ON, Canada \\ \{smithgm $\mid$ teather $\mid$ lassjw | carette\}@mcmaster.ca
}

\begin{abstract}
We present two experiments on large tiled displays used for fast-action gaming. Using a simulated tiled display, we assess the effects of interior bezel size, and configuration in a topdown arcade style space shooter game with many moving objects. Our experiments also investigate the effect of bezel compensation. The first experiment examines bezel size independent of configuration, using simulated bezel sizes ranging from 0 (i.e., no bezels) to $4 \mathrm{~cm}$ in a $3 \times 3$ grid configuration. Results of the experiment indicate minimal effects for bezel size and compensation. The second experiment fixed bezel size at $4 \mathrm{~cm}$ and instead varies configuration from a single display up to a 3x3 grid of simulated displays. Results of this study indicate that while the $1 \times 2$ performed worse in certain metrics, globally, the effects of configuration were similarly small.
\end{abstract}

Keywords — Large tiled displays; bezels; games

\section{INTRODUCTION}

Hardware trends have yielded ever cheaper and larger displays, and multi-output graphics cards. It is now feasible for consumers to build large multi-monitor (tiled) displays at home. Although home computers are used for various applications (e.g., work, school) they are becoming more commonly used for video games. Games are an area where large displays are relatively under-explored. Consequently, we argue that this is a ripe area for further research.

Game enthusiasts have certainly recognized the potential (and falling costs) of tiled multi-monitor configurations. Such displays are a recent trend in the game community. While generally more cost-effective than a single large display panel, multi-monitor configurations introduce other problems. For example, differences in brightness and colour between tiled monitors necessitate calibration of the display. A bigger problem is the gaps in the displayed image due to bezels - the borders of the individual monitors in a tiled-display configuration. These are annoying and distract players even as their task management and spatial awareness improves [1].

Display manufacturers have noticed the demand for multimonitor displays and strive to produce ever-smaller bezels to better resemble a single monitor display. Hardware manufacturers, notably NVidia and AMD, have also developed bezel compensation techniques intended to offset potential negative effects of breaking a displayed image across multiple monitor bezels. Typically, this takes the form of so-called
"French window" correction, which treats space behind the bezels as part of the coordinate space, much like the borders on a French door that occlude what is behind it [2]. Fig. 1 depicts examples of bezel compensation. Bezel compensation avoids the problems of "stretching" content across the bezels and prevents altering/warping cross-bezel trajectories. However, it is unclear if this alternative - which occludes content behind bezels - is actually preferable to stretching and warping, especially for games where small objects may be hidden impacting player performance.

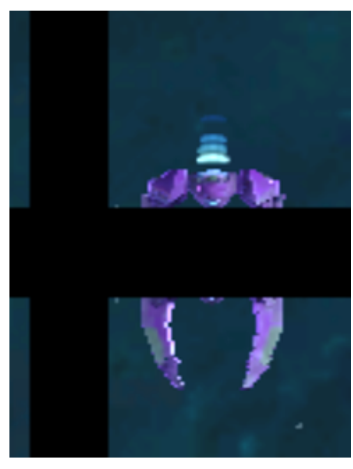

(a)

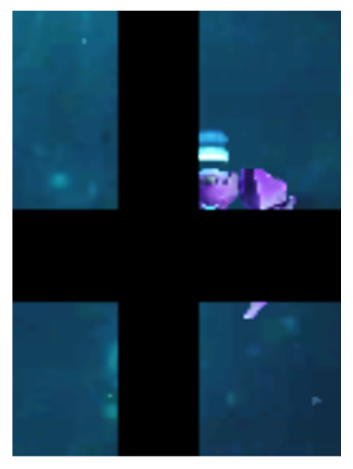

(b)

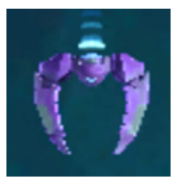

(c)
Fig. 1. Bezel compensation demonstrated. (a) depicts an enemy spaceship split across a horizontal bezel when bezel compensation is disabled. (b) depicts the ship occluded by bezels when bezel compensation is enabled. (c) depicts the ship unaffected by bezels for reference. Black bars represent our simulated monitor bezels.

Our work focuses on evaluating the impact of bezels have on game playing performance and enjoyment. Recent work [3, 4] has explored the effect of bezel size and configuration on largely static tasks such as visual search [3-5], size judgment [3], or on more interactive tasks such as point selection $[3,5$, 6]. To date, there appear to be no studies evaluating performance in a highly dynamic task like an action game. Given that this is a prevalent application area of tiled displays, we present a study investigating the effects of bezels on large displays used for gaming.

Our study is the first highly controlled experiment on bezel size and configuration in gaming. The only previous study addressing gaming on tiled displays [7] did not isolate these factors and used a qualitative approach. In particular, Sabri et al. [7] were primarily concerned with how participants used 
tiled displays in strategy games. Our concern is how well they can use them; hence we focus on user performance. We present two experiments. The first focuses on bezel size, using a fixed (simulated) display configuration. The second focuses on display configuration, using a fixed (simulated) bezel size.

\section{BACKGROUND}

Large displays in general [6, 8-11] and tiled displays specifically $[1,3-5,12,13]$ have been widely studied by human-computer interaction (HCI) researchers. We discuss relevant literature addressing both topics.

\section{A. Large Displays}

While many users find large displays more comfortable and more productive [8-10], there are still several usability issues preventing widespread adoption of large display setups. These problems include losing the cursor $[6,9,10]$, bezel problems across multiple displays $[2-5,10,12,13]$, distal information access [10], window management [10, 14], task management $[1,10,12,14]$, and configuration [10]. In video games, several of these problems are already adequately accounted for. For example, games rarely require extensive window management since they typically fill the entire screen and task management is limited to in-game activities.

Losing the cursor is a common problem for large display users due to forgetfulness when leaving their workstation or because they moved the cursor too quickly to compensate for large distances [6]. These problems are likely less prevelant in games, which often do not use traditional cursors. Instead, the player's attention is focused either on an avatar in third-person view games (e.g., Super Mario) or on a crosshair in first-person games such as Call of Duty. In the former, the player constantly attends to their avatar and is unlikely to "lose" it. In the latter, the crosshair is always fixed in the screen centre. Games that do require "traditional" cursor control (e.g., realtime strategy games) often employ hot keys, reducing reliance on the cursor and hence reducing the need to search for it on the large display. Consequently, games played on large displays may avoid this issue entirely.

Large displays enhance immersion in virtual environments [15], which may, in turn, help improve the sense of presence the feeling of "being there" - within a virtual environment [11]. In contrast, in exocentric views, users feel that the environment is rotating around them via panning and zooming. Egocentric views help users complete spatial tasks more accurately and quickly because they are more like real life [11]. Large displays support physical navigation as users can move their bodies to change their viewpoint $[12,16]$. Ball and North [12] suggest that large displays (both single screen and multimonitor tiled displays) lighten cognitive load and allow users to focus on their current task. Although physical navigation schemes are rare in games, the enhanced spatial awareness and reduced cognitive load offered by large displays may improve player performance, and yield a better overall experience.

\section{B. Tiled Displays}

With the falling cost and rising resolution of LCD panels, tiled displays are now a practical way of constructing extremely large high-resolution screens. Multiple monitor tiled displays are thus a good alternative to a single large display. It allows users to see the game world in both focus and periphery displays while reducing the number of actions that they need in order to change their field of view [14]. Compared to a single display, however, tiled displays generally require a calibration process to ensure consistency between each panel. This avoids differences in various factors between monitors including colour, refresh rate, and brightness levels [2].

Recent work indicates that wide interior bezels have a small negative effect on magnitude judgements for stationary objects. These errors are more noticeable when interactions are performed at arm's length. Wallace et al. [3] report that judgement errors increase by around 5\% when interior bezels are present for stationary objects. Task completion times also increase. Bezel compensation techniques appear to have little or no effect. Magnitude judgements generally become more difficult when the object is moving, so it would not be surprising if the judgement errors became worse. Our work is motivated by the suspicion that bezels will have a much greater impact in video games, where there are often multiple moving objects that players must keep track of.

The presence of interior bezels does not affect visual search times for stationary objects $[4,5]$, but error rates are increased when the target object is split across a bezel [5]. However, Wallace et al. [4] report contradictory results: objects that were split across bezels were actually easier to find, possibly due to participants employing different visual search strategies in these cases. Steering tasks are also negatively affected by bezels. Users tend to slow down upon reaching a bezel, then speed up after crossing it [5]. This can be especially detrimental in games where players must perform quick visual searches to find hazards, while simultaneously avoiding other obstacles. Target selection tasks are not affected by bezels, however. Users tend to perform coarse navigation while travelling to the object and switch to fine navigation when they are ready to select the object [5]. Typically, they cross the bezel during coarse ballistic motion. Many games incorporate pointing tasks (e.g., selecting a unit in a real-time strategy game, or targeting an enemy in a first-person shooter [17]), so these results may apply to games as well.

Researchers are currently investigating techniques to view information hidden behind bezels. One technique allows users to pan the image using gestural input, allowing them to temporarily move a section of the image out from behind a bezel by panning the entire image [2]. Another technique uses the user's physical location and head movement to pan the image as if they were looking out of a window. This technique can be difficult to control because it depends on the user's movement - keeping the image steady requires that the user remain still [2]. Moreover, these techniques require additional specialized tracking hardware, which average consumers generally cannot afford. Bezel issues can instead be mitigated by choosing thin-bezel screens. Multiple screens placed at different depths appear to be more detrimental to task performance than bezels [11], so organizing multiple monitors to form a plane or curve might help reduce some of the problems associated with bezel presence. 


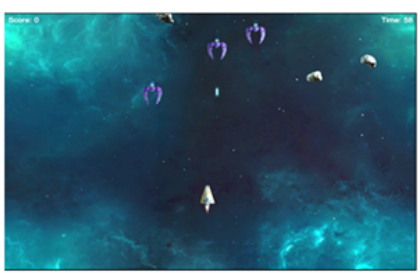

(a)

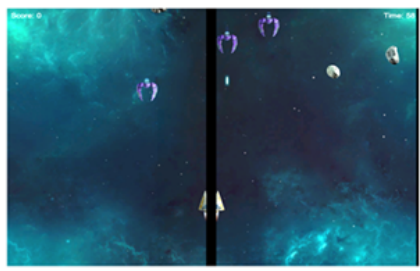

(e)

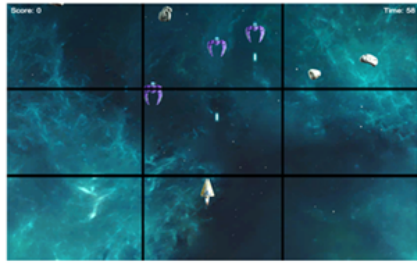

(b)

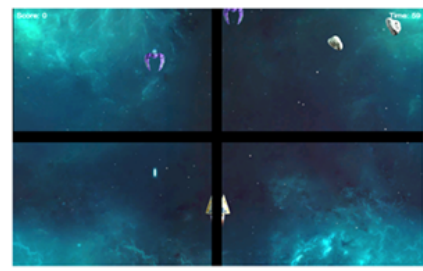

(f)

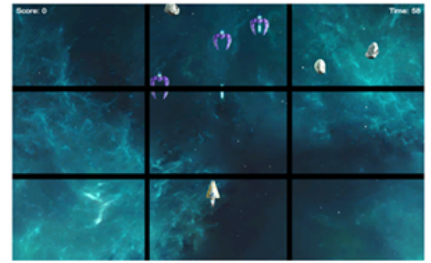

(c)

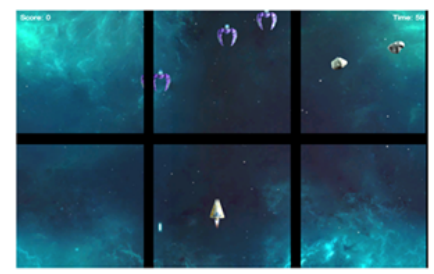

(g)

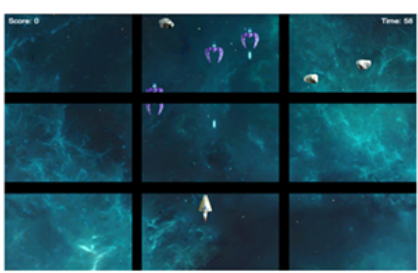

(d)

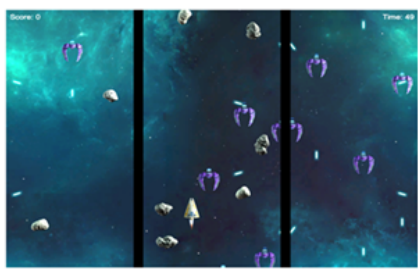

(h)

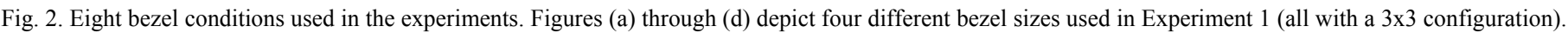
Figures (e) through (h) depict four configurations used in Experiment 2 (all with $4 \mathrm{~cm}$ bezel size). (a) " $0 \mathrm{~cm}$ ", i.e., no bezels basesline condition; (b) $1 \mathrm{~cm}$ bezels; (c) $2 \mathrm{~cm}$ bezels; (d) $4 \mathrm{~cm}$ bezels; (e) 1x2 configuration; (f) $2 \times 2$ configuration; (g) $2 \times 3$ configuration; (h) 1x3 configuration. All are shown with bezel compensation disabled. Not shown: $0.25 \mathrm{~cm}$ and $0.5 \mathrm{~cm}$ bezel conditions, and compensated bezels.

Another option to compensate for bezels in pointing tasks is using special cursor translation functions [6]. Cursor translation can occur across the virtual space, where bezels are ignored by the computer, or across physical space, where the computer compensates for the bezels. Movement in the virtual space is faster because there is less distance between points and many users found this preferable to the accurate representation of distance provided by the bezel compensated configuration [6].

\section{Methodology}

This section describes first the methodology common to both experiments, namely the apparatus and the procedure. Details specific to each experiment (participants, design) appear at the end of the section. Although our overall goal was to investigate the design space around bezel configuration, size, and compensation, we opted to investigate these factors in two separate experiments. The reason was that looking at several possible configurations and sizes in tandem (along with compensation) simply yields too many experimental conditions. Any single experiment looking at as many combined conditions would be difficult from a practical point of view (e.g., scheduling/recruiting participants). Future work will address this issue by picking a subset of specific combinations of configuration and bezel size for further study.

\section{A. Apparatus}

\section{1) Software Setup}

For the experiment, we developed a top-down space shooter game using Unity. This was based on the Unity Space Shooter tutorial ${ }^{1}$. Although we are also interested in user experience with commercial games, based on the arguments of other authors [18, 19] we favour using custom-developed games due to the relatively higher degree of experimental control provided. Fig. 2 depicts the game used in our study. The game involved controlling a player ship while avoiding asteroids, enemy ships, and projectiles (bullets) fired by

\footnotetext{
${ }^{1}$ See http://unity3d.com/learn/tutorials/projects/space-shooter.
}

enemies at the player. Enemy ships and asteroids both moved straight down from the top of the screen toward the bottom. Enemy bullets fired at regular intervals in straight lines or circular patterns with $50 \%$ probability.

Asteroids and enemy ships could be destroyed by a single shot from the player's gun. Their score would increase by 10 points for each asteroid destroyed, and 20 points for each ship. Bullets could also be shot down, but did not increase the player score. Similarly, the player was destroyed upon being hit by an enemy, asteroid, or enemy bullet. The player had unlimited lives, but the number of times they were destroyed by each obstacle was counted as a dependent variable (see design sections below).

The main modification to the software was to incorporate artificial bezels. The bezels were simulated in software, much like other recent research $[3,4]$. The main advantage of this approach is that it avoids display calibration issues and the requirement for multiple large monitors. The software allowed control of the width and number of bezels displayed. Bezel size could be as large as $4 \mathrm{~cm}$ or as a small as $0 \mathrm{~cm}$ (i.e., no bezels). Bezels could also be configured to simulate a single panel display (i.e., no bezels), and several other tiled display configurations, including $1 \times 2,1 \times 3,2 \times 2,2 \times 3$, and $3 \times 3$, where the first number indicates how many simulated displays were "vertically stacked", then side-by-side (i.e., 1x2 indicates two displays side-by-side, a common configuration). The software also supported simulation of bezel compensation, as described earlier (see also Fig. 1).

In conditions with bezel compensation, bezels were implemented simply by drawing black rectangles over the display corresponding to the specified bezel configuration/size combination. This yielded the desired effect: the coordinate space included the space under/behind the bezel, hence it was possible for a ship to "hide" behind these. This yields the same effect as the French window correction [2] described earlier. For conditions without bezel compensation, bezels were 
implemented as breaks in the coordinate system. Technically, this required displaying the scene using multiple virtual cameras (one for each "tile" in the configuration). All cameras were positioned the same distance from the scene to ensure that all objects retained their correct size across all virtual displays. However, the edges of the camera viewing volumes were adjusted to create gaps between them. The result effectively simulated standard multi-monitor setups without bezel compensation: an object moving across a bezel would immediately continue on the other side of the virtual bezel due to the break in the coordinates.

Note that in both experiments, one condition was duplicated: the single display (i.e., no bezels) was implemented both with and without bezel compensation. This was included to account for possible software performance (rather than human performance) differences due to implementation differences between the two bezel compensation conditions. In the single display without compensation, a $3 \times 3$ grid of virtual cameras were set up such that there was no space between their viewports. In the single display with compensation, a single virtual camera was used, similar to the rest of the compensated conditions that simply drew black bars over the display to represent bezels. The rationale for duplicating this condition was that although they visually appear the same, rendering multiple virtual cameras might be computationally more intensive. If this were the case, we would statistically detect such a difference and know that it may apply to any differences between other conditions too.

The software presented summary screens at the end of each trial. This gave participants an idea of how well they did on the completed trial. Their score and remaining time were always displayed during the trial. The software automatically proceeded through each condition in a counterbalanced order.

\section{2) Hardware Setup}

The experiment was conducted on a desktop PC with a 3.4 $\mathrm{GHz}$ quad-core Intel Core $\mathrm{i}$ processor. The computer had 8 GB of RAM, and was running the Windows 7 OS. Participants played the game using an Xbox One controller connected to the PC via USB. A 75 in. Samsung Series 77100 Smart TV (1920 x 1080 pixel resolution) was used for all conditions. The display was set to run in game mode to minimize input latency. We did not measure latency, as it was consistent across all conditions. Participants sat approximately $10 \mathrm{ft}$. from the display. The hardware setup can be seen in Fig. 3 .

\section{B. Procedure}

Upon arrival, the experimenter explained the purpose of the experiment and conditions while demonstrating the game. Participants gave informed consent before continuing. Following this introduction, participants were seated on the chair in a mock living-room setup (Fig. 3). The rules of the game were explained and then they were given the controller. Participants were instructed to play the game to the best of their ability. This involved staying alive by avoiding enemies and bullets for as long as possible while destroying as many enemies to increase their score. No game strategies or hints were given by the experimenter.

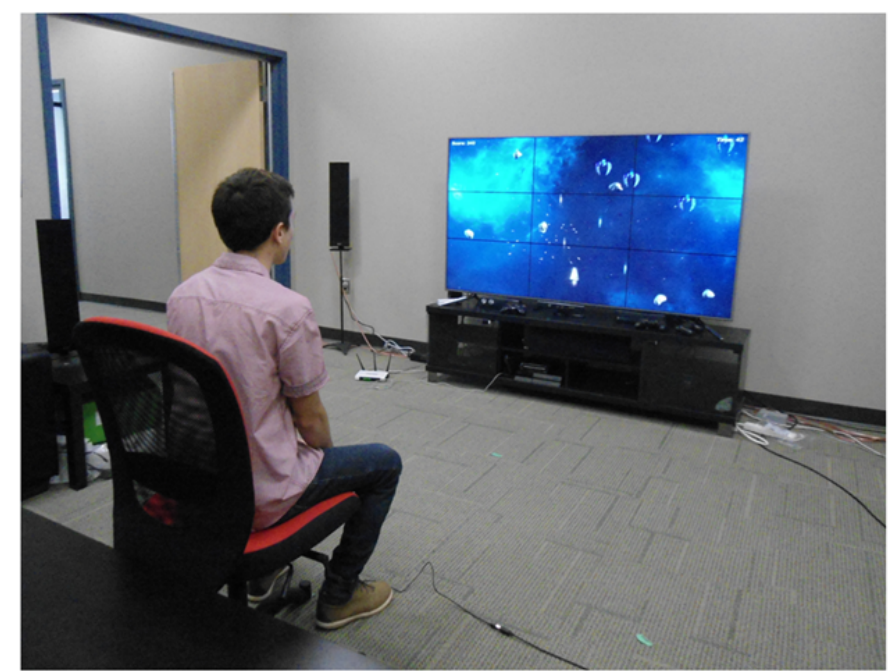

Fig. 3. Hardware setup showing a participant playing the game with $3 \times 3$ configuration and $0.5 \mathrm{~cm}$ bezels.

Each participant played four one-minute trials in each condition for the experiment they participated in (either Experiment 1, or Experiment 2, see below). At the end of a trial, the participant was given a summary of their performance (e.g., longest survival time, how many times they were destroyed, number of enemies destroyed, etc.). They were prompted to press the left bumper button to progress, and thus could take a break between trials. Upon completing the experiment, participants filled out a questionnaire about the conditions and to gather demographics.

\section{Experiment 1}

\section{1) Participants}

Eighteen participants took part in the study. Their ages ranged from 18 to 30 years, with a mean age of $22.2(S D=3.4)$ years. Twelve were male and 16 were right-handed. All participants had normal or corrected-to-normal vision.

Participants were asked about their game habits as this may influence their performance. We asked how frequently they played computer games (with mouse and keyboard), console games (with a gamepad) and mobile games (e.g., smartphone or tablet). Participant game playing is summarized in Fig. 4. "Rarely" indicates they play once or twice per month, "sometimes" indicates several times per month, and "frequently" indicates they play several times per week.

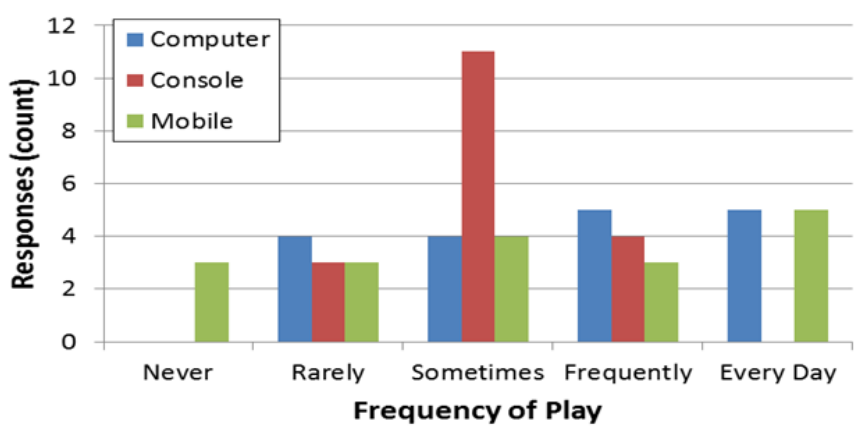

Fig. 4. Experiment 1 participant gaming habits histogram by game type and play frequency. 


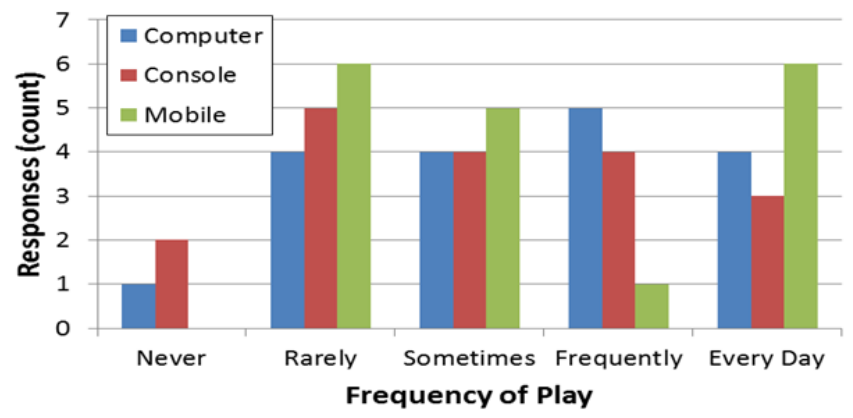

Fig. 5. Experiment 2 participant gaming habits histogram by game type and play frequency

\section{2) Design}

The experiment used a within-subjects design. The independent variables and their levels were:

Size: $0 \mathrm{~cm}, 0.25 \mathrm{~cm}, 0.5 \mathrm{~cm}, 1 \mathrm{~cm}, 2 \mathrm{~cm}, 4 \mathrm{~cm}$

Compensation: on, off

Trial: 1, 2, 3, 4

Size indicates the width of the bezels. The 12 combinations of size and compensation were counterbalanced according to a balanced Latin square. Monitor configuration was held constant at $3 \times 3$, as it was expected that the higher number of bezels would elicit a stronger response. This corresponds to a condition in Experiment 2, below. Each participant played 48 one-minute trials for this experiment. Over all 18 participants, this yielded a total of 864 one minute trials.

The dependent variables were ship kill ratio (SKR), asteroid kill ratio (AKR), bullet kill ratio (BKR), ship and asteroid deaths (SAD), bullet deaths (BD), and longest streak. Each "kill ratio" was the percentage of that enemy type that were destroyed by the player. Each "death" type was the count of how many times the player was killed by that enemy type. Longest streak was the longest duration (in seconds) in a given trial that the player stayed alive for.

\section{Experiment 2}

\section{1) Participants}

Eighteen participants took part in the study. Of these, two of the participants also took part in Experiment 1. Their ages ranged from 18 to 52 years, with a mean age of $23.5(S D=8.8)$ years. Eleven were male and all were right-handed. All participants had normal or corrected-to-normal vision. Game playing habits were recorded in the same fashion as in Experiment 1 and summarized in Fig. 5.

\section{2) Design}

The experiment used a within-subjects design. The independent variables and their levels were:

Configuration: $1 \times 1,1 \times 2,1 \times 3,2 \times 2,2 \times 3$, and $3 \times 3$

Compensation: on, off

Trial: 1, 2, 3, 4

The 12 combinations of configuration and correction were counterbalanced according to a balanced Latin square. Participants played a total of 48 games for this experiment. Like Experiment 1, this yielded a total of 864 one minute trials over all participants. Bezel size was held constant at $4 \mathrm{~cm}$ to match our physical monitors, and to corresponding to the $4 \mathrm{~cm}$ condition in Experiment 1. This experiment used the same dependent variables as experiment 1 .

\section{RESULTS AND DISCUSSION}

Results from both experiments are detailed in this section. Each dependent variable was analyzed with repeated-measures ANOVA. Statistical reports are shown in Tables I and II.

\section{A. Experiment 1}

We first analyze longest streak, then look at enemy kill ratios and player deaths. Statistical results are shown in Table I. As can be seen in Table I, there were no significant effects on longest streak. The grand mean longest streak was $19.2 \mathrm{~s}$. The difference due to bezel compensation was miniscule: $19.3 \mathrm{~s}$ with compensation on vs. $19.1 \mathrm{~s}$ with it disabled. No single condition was greater than $10 \%$ different from the mean, with values ranging from $17.9 \mathrm{~s}$ to $21.4 \mathrm{~s}$. Overall, any effect due to bezel size or compensation on longest streak was minimal.

\section{1) Kill Ratios}

Since there were three classes of enemies (ships, asteroids, and bullets), we counted kill ratios (and deaths) separately. The rationale was that smaller objects, e.g., bullets, may be affected differently by bezel size than larger objects. Results for each kill ratio (averaged over all trials) are shown in Fig. 6 due to space constraints.

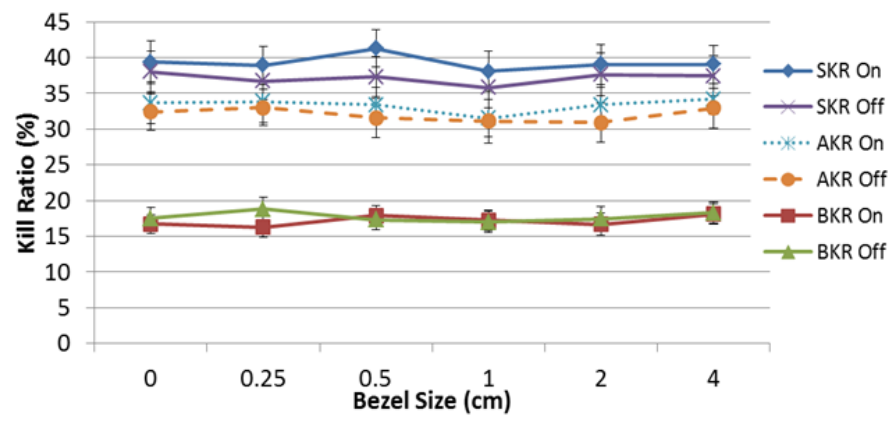

Fig. 6. Experiment 1 kill ratio dependent variables as a function of bezel size. Specific metrics indicated in legend. Conditions both with and without compensation shown, indicated by "On" and "Off". Error bars indicate $95 \%$ confidence intervals.

Interestingly, and as seen in,Fig. 6 the effect of bezel compensation was very small, and was not significant for any of the kill ratio dependent variables. Similarly, bezel size had little effect, with the exception of the significant interaction effect between bezel size and trial for bullet kill ratio (see Table I). Tukey-Kramer posthoc analysis $(p<.05)$ revealed that participants did not improve with trial with $4 \mathrm{~cm}$ bezel, but improved significantly with $0 \mathrm{~cm}$ bezels by the $4^{\text {th }}$ trial.

\section{2) Player Deaths}

Since enemy ships and asteroids moved similarly, and were roughly the same size, we summed player deaths due to being hit by either of these obstacles into a single dependent variable, ship and asteroid deaths (SAD). Since there was a greater number of bullets (spawned by ships) and they moved differently, we counted deaths due to bullets separately as bullet deaths (BD). See Fig. 7. 
TABLE I. EXPERIMENT 1 STATISTICAL EFFECTS. * INDICATES SIGNIFICANT RESULT, $P<.05 . * *$ INDICATES SIGNIFICANT RESULT, $P<.01$.

\begin{tabular}{|c|c|c|c|c|c|c|c|c|c|c|c|c|c|}
\hline \multirow[t]{2}{*}{ Effect } & \multirow[t]{2}{*}{ d. $f}$. & \multicolumn{2}{|c|}{$S K R$} & \multicolumn{2}{|c|}{$A K R$} & \multicolumn{2}{|c|}{$B K R$} & \multicolumn{2}{|c|}{$S A D$} & \multicolumn{2}{|c|}{$B D$} & \multicolumn{2}{|c|}{ Longest Streak } \\
\hline & & $F$ & $p$ & $F$ & $p$ & $F$ & $p$ & $F$ & $p$ & $F$ & $p$ & $F$ & $p$ \\
\hline (S)ize & 5,17 & 1.07 & .38 & 2.36 & $*$ & 0.98 & ns & 1.59 & .17 & 0.47 & ns & 1.54 & .19 \\
\hline (C)ompensation & 1,17 & 1.54 & .23 & 1.17 & .29 & 0.27 & ns & 0.49 & ns & 0.02 & ns & 0.01 & ns \\
\hline (T)rial & 3,17 & 1.40 & .25 & 1.57 & .21 & 3.08 & $*$ & 0.71 & $\mathrm{~ns}$ & 4.75 & $* *$ & 1.85 & 0.15 \\
\hline$S \times C$ & 5,85 & 0.39 & ns & 0.31 & ns & 1.87 & .10 & 1.01 & .41 & 1.81 & .11 & 0.61 & ns \\
\hline$S \times T$ & 15,85 & 0.98 & ns & 1.01 & .44 & 2.05 & $*$ & 0.62 & $\mathrm{~ns}$ & 1.65 & .06 & 1.16 & .31 \\
\hline$C \times T$ & 3,85 & 0.54 & ns & 0.15 & ns & 2.05 & .11 & 0.73 & ns & 1.89 & .14 & 1.32 & .28 \\
\hline$S \times C \times T$ & 15,255 & 1.02 & .43 & 0.76 & ns & 1.01 & .44 & 1.20 & .27 & 0.71 & ns & 0.78 & $\mathrm{~ns}$ \\
\hline
\end{tabular}

TABLE II. EXPERIMENT 2 STATISTICAL EFFECTS. * INDICATES SIGNIFICANT RESULT, $P<.05 . * *$ INDICATES SIGNIFICANT RESULT, $P<.01$.

\begin{tabular}{|c|c|c|c|c|c|c|c|c|c|c|c|c|c|}
\hline \multirow{2}{*}{ Effect } & \multirow[t]{2}{*}{ d. $f}$. & \multicolumn{2}{|c|}{$S K R$} & \multicolumn{2}{|c|}{$\overline{A K R}$} & \multicolumn{2}{|c|}{$B K R$} & \multicolumn{2}{|c|}{$S A D$} & \multicolumn{2}{|c|}{$B D$} & \multicolumn{2}{|c|}{ Longest Streak } \\
\hline & & $F$ & $\bar{p}$ & $F$ & $p$ & $F$ & $\bar{p}$ & $F$ & $p$ & $F$ & $p$ & $F$ & $\bar{p}$ \\
\hline$C(O)$ nfiguration & 5,17 & 3.49 & $* *$ & 0.51 & ns & 1.17 & .33 & 1.02 & .41 & 0.58 & ns & 1.41 & .23 \\
\hline (C)ompensation & 1,17 & 0.07 & ns & 0.89 & ns & 1.24 & .28 & 3.62 & .07 & 0.64 & $\mathrm{~ns}$ & 0.00 & $\mathrm{~ns}$ \\
\hline (T)rial & 3,17 & 2.37 & .08 & 1.01 & .39 & 0.68 & ns & 0.80 & ns & 0.28 & ns & 1.93 & .14 \\
\hline$O \times C$ & 5,85 & 1.15 & .34 & 0.82 & ns & 0.74 & ns & 1.25 & .29 & 0.27 & ns & 1.19 & .32 \\
\hline$O \times T$ & 15,85 & 1.16 & .31 & 0.97 & ns & 1.11 & .34 & 1.40 & .15 & 0.74 & ns & 0.73 & $\mathrm{~ns}$ \\
\hline$C \times T$ & 3,85 & 1.65 & .19 & 0.81 & ns & 2.30 & .09 & 1.46 & .24 & 2.07 & .11 & 0.85 & $\mathrm{~ns}$ \\
\hline$O \times C \times T$ & 15,255 & 1.47 & .12 & 1.02 & .43 & 0.90 & ns & 0.83 & ns & 0.67 & ns & 0.71 & $\mathrm{~ns}$ \\
\hline
\end{tabular}

As noted in Table I, none of the conditions significantly affected ship and asteroid deaths. This is further reflected by the flat lines across bezel size and wide confidence intervals demonstrated in Fig. 7. This is perhaps unsurprising: the size of these objects generally prevented them from being fully occluded in conditions with bezel compensation enabled. When bezel compensation was disabled, they would be fairly easy to keep track of, due to being stretched across bezels.

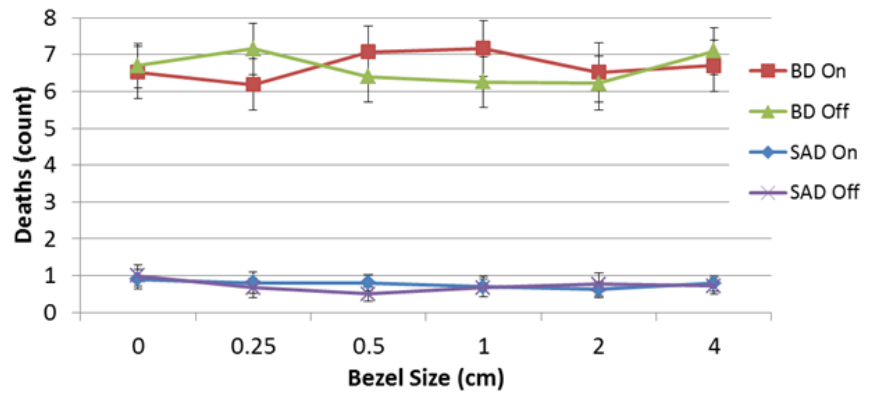

Fig. 7. Experiment 1 ship and asteroid deaths and bullet deaths as a function of bezel size. Specific metrics indicated in legend. Conditions both with and without compensation shown (indicated by "On" and "Off"). Error bars show $95 \%$ confidence intervals.

The only significant effect for bullet deaths was the main effect trial. Globally, participants tended to get hit by bullets less often in later trials, but the absence of interaction effects suggests that this rate of improvement did not change more or less quickly with any particular bezel condition.

\section{1) Subjective Results}

We also asked participants to rank their experience with each bezel type and each level of bezel compensation. Specifically, they were asked "Which bezel size did you feel it was easiest to play with/without compensation?". Participants overwhelmingly preferred "thinnest" bezels $(0.25,0.5 \mathrm{~cm})$, regardless of the state of bezel compensation. See Fig. 8.
When asked "Do you prefer to play with or without bezel compensation?" the results were less clear: of the 18 participants, 7 preferred playing with compensation, 5 preferred playing without compensation, and 6 felt it didn't matter. Interestingly, this suggests that participants were also aware of the negligible performance difference yielded by bezel compensation, as reported in the results above.

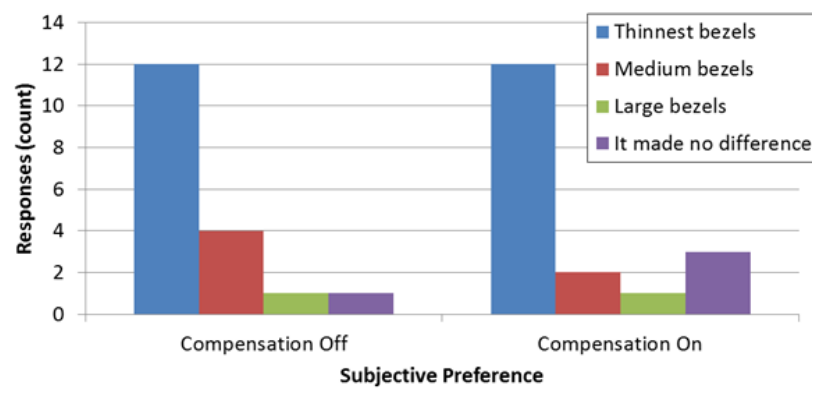

Fig. 8. Experiment 1 histogram of participant subjective preference responses.

\section{B. Experiment 2}

We report our experiment 2 results in the same fashion as experiment 1 , starting with longest streak then moving onto kill ratios and player deaths. Statistical results are in Table II. None of the independent variables nor their interaction effects significantly affected longest streak (see Table II). Most of these effects were again very small. The grand mean for longest streak was $13.8 \mathrm{~s}$, substantially shorter than in Experiment 1 (19.3 s average). This may be due to differences in the participant pool, e.g., game playing experience. Similar to experiment 1 though, there was little variability (less than $10 \%$ difference from the mean) in longest streak between conditions. These scores ranged from $12.6 \mathrm{~s}$ to $15.3 \mathrm{~s}$.

\section{1) Kill Ratios}

In terms of ship kill ratio (SKR), there was a significant main effect for configuration (only), see Table II. Tukey- 
Kramer posthoc analysis revealed that the $1 \times 2$ configuration was significantly worse than either of the single display (i.e., $1 \times 1$ ) or the $1 \times 3$ configuration (i.e., 3 adjacent displays). This is apparent in the top two lines (SKR On and SKR Off) for the ship kill ratio metric in Fig. 9. None of the other individual conditions were significantly different from one another.

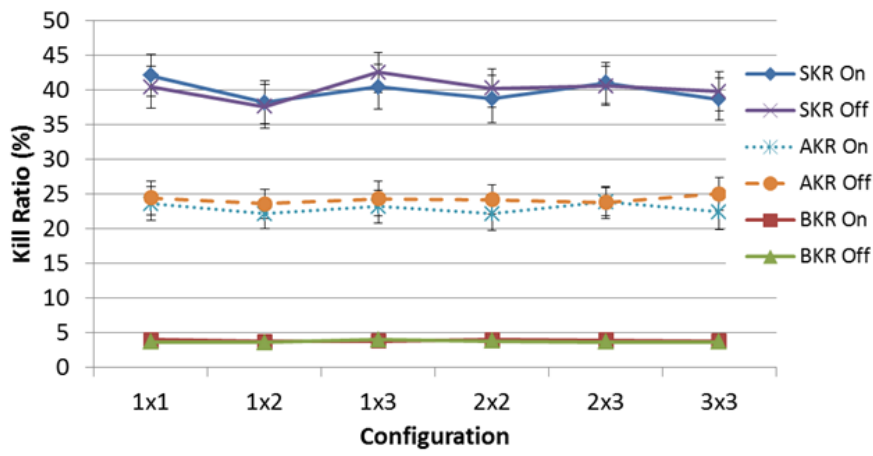

Fig. 9. Experiment 2 kill ratio dependent variables as a function of bezel size. Specific metrics indicated in legend. Conditions both with and without compensation shown, indicated by "On" and "Off". Error bars indicate $95 \%$ confidence intervals.

For asteroid kill ratio and bullet kill ratio, none of the main effects nor their interactions were significant, see Table II. Indeed, this is largely evident in the relatively flat lines for AKR and especially BKR (both on and off) in Fig. 9. In fact, there was virtually no difference at all in bullet kill ratio due to bezel compensation, as the lines for compensation on and off have nearly identical values for each configuration.

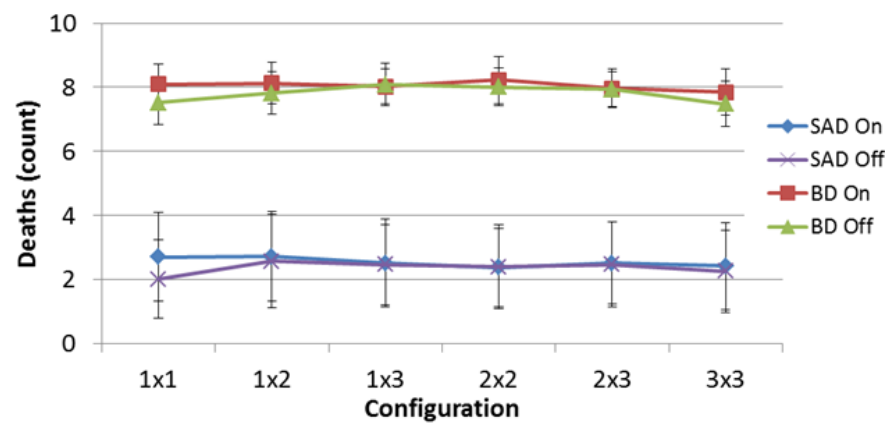

Fig. 10. Experiment 2 ship and asteroid deaths and bullet deaths as a function of bezel size. Specific metrics indicated in legend. Conditions both with and without compensation shown (indicated by "On" and "Off"). Error bars show $95 \%$ confidence intervals.

Overall, similar to experiment 1 , there were minimal effects for our independent variables in experiment 2. The most substantial (and only significant) effect noted was due to the configuration, and only on one of these dependent variables. Specifically, the main difference noted was due to the presence of the bezel down the center of the tiled display, which only occurred with the $1 \times 2$ configuration. This is interesting, although perhaps not surprising, and has implications for developers targeting multi-monitor setups, as this is likely the most commonly used home display configuration (as it would only require two monitors). Gamers intending to use a multimonitor setup at home might also consider getting three displays rather than two. We expect this effect may also be pronounced in poorly designed first-person shooter FPS games where the bezel cuts down the center of the display (where the targeting reticule is most commonly located).

\section{2) Player Deaths}

Similar to the other dependent variables, player deaths (both in terms of ship and asteroid deaths, and bullet deaths) were hardly affected by the conditions of interest. None of the independent variables significantly affected either ship and asteroid deaths or bullet deaths (see Table II).

Like most of our dependent variables, the lines in Fig. 10 indicate minimal differences in the scores for SAD and BD, between bezel compensation on or off, or across the different configurations. This is somewhat surprising, as the impact of configuration was expected to be stronger than bezel size. Nevertheless, our data indicates that even the $1 \times 2$ condition (which was significantly worse than $1 \times 1$ or $1 \times 3$ for SKR) did not offer worse performance than any other condition here.

\section{3) Subjective Results}

Like experiment 1, we surveyed participants about their subjective experience following the experiment. Specifically, they were asked "Which condition did you find easiest to play with/without compensation?". Responses were grouped into "fewest bezels $(1 \times 2,2 \times 2)$ ", "medium number of bezels $(1 \times 3)$ ", and "many bezels $(2 \times 3,3 \times 3)$ ". See Fig. 11 . With compensation off, participants preferred a medium number of bezels. Like experiment 1, we also asked their preference regarding compensation. Of the 18 participants, 9 preferred compensation on, 6 preferred it off, and 3 were undecided.

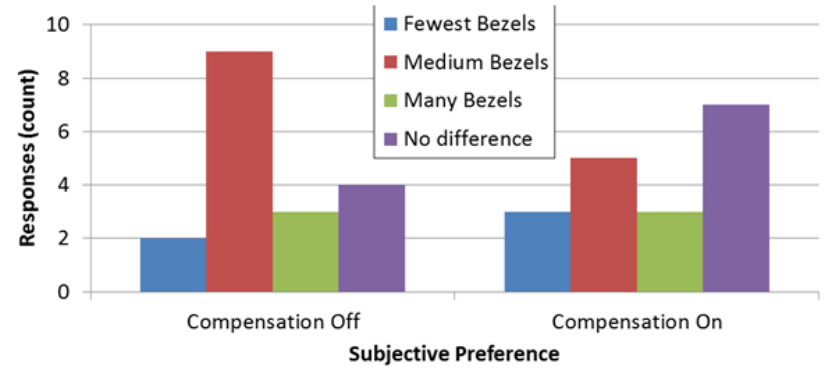

Fig. 11. Experiment 2 histogram of participant subjective preference responses.

\section{DISCUSSION}

It is surprising that our experiments did not detect differences between our conditions of interest. We had expected that due to the highly dynamic nature of our game task, the impact of bezels might be stronger than in previous work. Instead, and consistent with previous work [3, 4, 5], we found minimal effects for bezel size, configuration, or compensation. In general, these factors did not impact performance significantly, and any differences observed were small. We note that this does not conclusively demonstrate that bezel size, configuration, and compensation do not affect player performance or experience, as it is not in the nature of statistical testing to "prove the null hypothesis" in this fashion.

The largest differences were the learning effects on the nobezel experiment $10 \mathrm{~cm}$ condition, and the $1 \times 2$ display configuration in experiment 2. The first result suggests that participants improved faster without bezels than with $4 \mathrm{~cm}$ 
bezels at shooting enemy bullets. In contrast, their ability to shoot bullets did not improve with $4 \mathrm{~cm}$ bezels over the four trials. This makes sense, as the bullets were small and could "hide" behind bezels when compensation was enabled, and might still be unnoticed when split across bezels without compensation. This suggests that there may be some merit to thinner bezels, but overall, this result is overshadowed by the fact that most dependent variables were consistently not significantly affected by bezel size or compensation.

The influence of the $1 \times 2$ display configuration on ship kill ratio in experiment 2 is also interesting. Again, this is likely the worst condition overall as the bezel splits the center of the display vertically down the middle. We note that the $2 \times 2$ configuration also has the same limitation, and while it was not significantly worse, it was slightly worse than the bestperforming conditions. However, the result is particularly important for the $1 \times 2$ configuration, which is a common multidisplay setup as it requires the user only purchase one extra monitor. We note here that many games rely on content centralized in the display. For example, first-person shooter games typically display the targeting reticule in the center of the screen. Hence we caution developers against naïve "splitting" of content across two displays.

\section{CONCLUSIONS}

We presented two user studies looking at the influence of bezels size and configuration on game playing. Our studies used a top-down space shooter. Overall, our results consistently show limited effect for bezel size, configuration, and compensation. While some significant effects were noted (notably subtle differences between no-bezel and wide bezel conditions, or between $1 \times 2$ and single display configurations) most of our results were not statistically significant. In a sense, this is somewhat encouraging that the effects of bezels may be reasonably small. It appears that developer efforts on bezel compensation techniques, and even thin-bezel monitors may be misspent, as these technologies do not appear to help gaming. In fact, the additional display real-estate offered by multimonitor setups more than likely far outweighs the small negative effect of bezels. That said, we caution readers that failure to detect significant effects does not conclusively prove there is no difference. Without further studies to confirm these results, we can currently only cautiously suggest that the negative impact of bezels may well be overstated.

\section{A. Future Work}

We plan to further investigate the effects of bezels in tileddisplay gaming. In particular, the effects of bezels may be highly task dependent. We chose the shooter game used in our study on the premise that small objects (e.g., bullets) may prove difficult to track in the presence of bezels, but apparently, this was less problematic than expected. In future work, we plan to explore this in different game genres, e.g., first-person shooter games, strategy games, and so on.

\section{ACKNOWLEDGEMENTS}

Thanks to Pat Bennet and Allison Sekuler for insights into the experiment design and conditions. This work was supported by Canada Foundation for Innovation.

\section{REFERENCES}

[1] R. Ball and C. North, "Analysis of user behavior on high-resolution tiled displays," in Human-Computer Interaction-INTERACT 2005, ed: Springer, 2005, pp. 350-363.

[2] R. A. De Almeida, C. Pillias, E. Pietriga, and P. Cubaud, "Looking behind bezels: French windows for wall displays," in Proceedings of the International Working Conference on Advanced Visual Interfaces, 2012, pp. 124-131.

[3] J. R. Wallace, D. Vogel, and E. Lank, "The effect of interior bezel presence and width on magnitude judgement," in Proceedings of Graphics Interface 2014, 2014, pp. 175-182.

[4] J. R. Wallace, D. Vogel, and E. Lank, "Effect of bezel presence and width on visual search," Proceedings of The International Symposium on Pervasive Displays, 2014, pp. 118-123.

[5] X. Bi, S.-H. Bae, and R. Balakrishnan, "Effects of interior bezels of tiled-monitor large displays on visual search, tunnel steering, and target selection," in Proceedings of the ACM Conference on Human Factors in Computing Systems - CHI 2010, 2010, pp. 65-74.

[6] M. A. Nacenta, R. L. Mandryk, and C. Gutwin, "Targeting across displayless space," in Proceedings of the ACM Conference on Human Factors in Computing Systems - CHI 2008, 2008, pp. 777-786.

[7] A. J. Sabri, R. G. Ball, A. Fabian, S. Bhatia, and C. North, "Highresolution gaming: Interfaces, notifications, and the user experience," Interacting with Computers, vol. 19, pp. 151-166, 2007.

[8] M. Czerwinski, G. Robertson, B. Meyers, G. Smith, D. Robbins, and D. Tan, "Large display research overview," in Extended Abstracts on Human Factors in Computing Systems - CHI EA 2006, 2006, pp. 69-74.

[9] M. Czerwinski, G. Smith, T. Regan, B. Meyers, G. Robertson, and G. Starkweather, "Toward characterizing the productivity benefits of very large displays," in Proceedings of INTERACT, 2003, pp. 9-16.

[10] G. Robertson, M. Czerwinski, P. Baudisch, B. Meyers, D. Robbins, G. Smith, and D. Tan, "The large-display user experience," IEEE Computer Graphics and Applications, vol. 25, pp. 44-51, 2005.

[11] D. S. Tan, D. Gergle, P. Scupelli, and R. Pausch, "With similar visual angles, larger displays improve spatial performance," in Proceedings of the ACM Conference on Human Factors in Computing Systems - CHI 2003, 2003, pp. 217-224.

[12] R. Ball and C. North, "Effects of tiled high-resolution display on basic visualization and navigation tasks," in Extended Abstracts on Human Factors in Computing Systems - CHI EA 2005, 2005, pp. 1196-1199.

[13] D. S. Tan and M. Czerwinski, "Effects of visual separation and physical discontinuities when distributing information across multiple displays," in Proceedings of Interact 2003, 2003, pp. 252-255.

[14] J. Grudin, "Partitioning digital worlds: focal and peripheral awareness in multiple monitor use," in Proceedings of the ACM Conference on Human Factors in Computing Systems - CHI 2001, 2001, pp. 458-465.

[15] T. Ni, D. A. Bowman, and J. Chen, "Increased display size and resolution improve task performance in information-rich virtual environments," in Proceedings of Graphics Interface 2006, 2006, pp. 139-146.

[16] D. S. Tan, D. Gergle, P. G. Scupelli, and R. Pausch, "Physically large displays improve path integration in 3D virtual navigation tasks," in Proceedings of the SIGCHI Conference on Human Factors in Computing Systems - CHI 2004, 2004, pp. 439-446.

[17] J. Looser, A. Cockburn, and J. Savage, "On the validity of using firstperson shooters for Fitts' law studies," British HCI Conference, 2005, pp. 33--36.

[18] R. P. McMahan, E. D. Ragan, A. Leal, R. J. Beaton, and D. A. Bowman, "Considerations for the use of commercial video games in controlled experiments," Entertainment Computing, vol. 2, pp. 3-9, 2011.

[19] R. J. Teather and I. S. MacKenzie, "Comparing order of control for tilt and touch games," Proceedings of the 2014 Conference on Interactive Entertainment, Newcastle, NSW, Australia, 2014, pp. 1-10. 